\title{
ON RISING SUN VON MISES AND RISING SUN WRAPPED CAUCHY CIRCULAR MODELS
}

\author{
A.J.V. RADHIKA, S.V.S.GIRIJA, A.V. DATTATREYA RAO
}

\begin{abstract}
By applying wrapping (Dattatreya Rao et al (2007)), offsetting (Girija et al (2013 and Radhika et al (2013)) and stereographic projection (Phani et al (2012)) have constructed circular models.

Further the circular models based on the Rising Sun function are motivated by purely mathematical considerations as a smoothing function and possible application. This work takes a further step in this direction using several mathematical tools such as Real Analysis along with MATLAB and are applied to enlarge the horizon of Mathematical Statistics. Here an attempt is made to construct new circular models using the Rising Sun function and also the circular rising sun lemma is proposed.
\end{abstract}

Mathematics Subject Classification 2000: 60E10

Keywords : Circular model, trigonometric moments, Rising Sun function, characteristic function, von Mises and Wrapped Cauchy circular models.

\section{INTRODUCTION}

The available methods of generating circular models are wrapping a linear model, offsetting a bivariate linear model and applying stereographic projection on a linear model. The Rising Sun function [Van Rooij and Schikoff (1982), p.10] smoothens the existing curve and many bumps disappear. This may lead to the effect of increasing the smoothing the curve in density estimation. Girija (2010) proposed a new method of generating circular model by using the Rising Sun function (RSF). Motivated by the mathematical significance of the Rising Sun function behind the construction of circular models, an attempt is made to construct new circular models and various properties are discussed. In this paper two circular models are constructed by applying the Rising Sun function and their population characteristics are computed numerically.

\section{CONSTRUCTION OF CIRCULAR MODELS USING THE RISING SUN FUNCTION}

The Rising Sun function (RSF) of a bounded function $f:[a, b] \rightarrow \mathrm{R}$ is defined by

$$
f_{\Theta}(x)=\sup \{f(t): x \leq t \leq b\}
$$


It is easy to show that

$>$ when $f$ is nonnegative then $f_{\Theta}$ is nonnegative

$>$ when $f$ is continuous then $f_{\Theta}$ is continuous

$>f_{\Theta}$ is monotonically decreasing, hence $f_{\Theta}=f$ when $f$ is decreasing and $f_{\Theta}$ is the smallest monotonically decreasing function such that $f_{\Theta}=f$.

Imagine the Rising Sun on $x$ - axis. Then $\left\{(x, y) \in \mathrm{R}^{2}: y \geq f_{\Theta}(x)\right\}$ is illuminated by the sun whereas $\left\{(x, y) \in \mathrm{R}^{2}: y<f_{\Theta}(x)\right\}$ is covered by darkness. The set $\left\{(x, f(x)): f(x)=f_{\Theta}(x)\right\}$ is the collection of those points of the graph of $f$ that receive light from the sun.

Theorem 2.1: [Van Rooij and Schikoff (1982)] If $f$ is continuous on $[a, b]$ then for any $k$ in the range of $f_{\Theta}, S=\left\{x \in[a, b]: f_{\Theta}(x)=k\right\}$ is a closed and bounded interval.

The Rising Sun Lemma 2.2: [Van Rooij and Schikoff (1982)]: Let $f:[a, b] \rightarrow \mathrm{R}$ be a continuous function. Then $E=\left\{x \in(a, b): f_{\Theta}(x)>f(x)\right\}$ is open. If $(\alpha, \beta)$ is a component of $E$ then $f_{\Theta}(\beta)=f(\beta)$ and $f_{\Theta}(\alpha)=f(\alpha)$ for $\alpha \neq a$, and $f_{\Theta}$ is constant in that interval.

The well known Lebesgue theorem is also proved by Van Rooij and Schikoff (1982) using the Rising Sun function.

A new construction procedure of a class of Circular Models using RSF is obtained in the following theorem and an illustration is also included in this section. These distributions are named as 'Rising Sun Circular models'.

Theorem 2.3: [Girija (2010)]: If $\mathrm{g}$ is the pdf and $G$ is the cdf of a random variable of a circular distribution then the Rising Sun function $g_{\Theta}$, gives rise to the pdf $g_{c}$ of a circular model. The distribution function of $g_{c}$ is given by 


$$
G_{c}= \begin{cases}\frac{1}{\mathrm{~K}}\left[\theta_{1} g\left(\theta_{1}\right)+G(\theta)-G\left(\theta_{1}\right)\right] & \text { for } \theta_{1}<\theta \\ \frac{1}{\mathrm{~K}}\left[\theta \mathrm{g}\left(\theta_{1}\right)\right] & \text { for } \theta_{1} \geq \theta\end{cases}
$$

Analogous to Theorem 2.2 the Rising Sun lemma for circular data is presented as follows

The Circular Rising Sun Lemma 2.4: Let $g:[0,2 \pi] \rightarrow \mathrm{R}$ be a continuous function. Then $E=\left\{\theta \in(0,2 \pi): g_{\Theta}(\theta)>g(\theta)\right\}$ is open. If $(\alpha, \beta)$ is a component of $E$ then $g_{\Theta}(\beta)=g(\beta)$ and $g_{\Theta}(\alpha)=g(\alpha)$ for $\alpha \neq 0$, and $g_{\Theta}$ is constant in that interval. $g_{c}$ is the normalized function of $g_{\Theta}$, hence $\beta$ represents the mode of the circular model at which both $g_{\Theta}$ and $g_{c}$ have maximum value. Hence component of $g_{c}$ as well as $g_{\Theta}$ is $(0, \beta)$ and $g_{c}$ is the pdf of circular model known as Rising Sun circular model.

\section{The Rising Sun von Mises Model}

The von Mises distribution was introduced by von Mises (1918) in order to study the deviations of measured atomic weights from integral values. A technique is proposed in which each of these microphone-pair determined azimuths are further combined into a mixture of the von Mises distributions, thus producing a practical probabilistic representation of the microphone array measurement. It is shown that this distribution is inherently multimodal and that the system at hand is non-linear, which required a discrete representation of the distribution function by means of particle filtering [Ivan Markovi'c, and Ivan Petrovi'c(2010)]. A multivariate extension of the bivariate model of Singh et al (2002) is proposed by Mardia et al (2008). Procedures for the estimation of parameters of the proposed distribution include the method of moments, and pseudolikelihood; the efficiency of the latter is investigated in two and three dimensions. The methods are applied to real protein data of conformational angles. 


\section{A.J.V. RADHIKA, S.V.S.GIRIJA, A.V. DATTATREYA RAO}

The pdf of von Mises distribution [Jammalamadaka and Sengupta (2001)] is

$$
g(\theta)=\frac{1}{2 \pi I_{0}(k)} \exp (k \cos (\theta-\mu)),
$$

where $I_{0}$ denotes the Modified Bessel function of the first kind and order zero, which can be defined by

$$
I_{0}(k)=\frac{1}{2 \pi} \int_{0}^{2 \pi} e^{k \cos \theta} d \theta .
$$

The function $\mathrm{I}_{0}$ has power series expansion

$$
I_{0}(k)=\sum_{r=0}^{\infty} \frac{1}{(r !)^{2}}\left(\frac{k}{2}\right)^{2 r}
$$

The parameter $\mu$ is the mean direction and the parameter $k$ is known as the concentration parameter. The mean resultant length $\rho$ is $A(k)$ where $A$ is the function defined by

$$
A(k)=\frac{I_{1}(k)}{I_{0}(k)}
$$

The Rising Sun function of the von Mises distribution is given by

$$
\begin{aligned}
g_{\Theta}(\theta) & =\operatorname{Sup}(g(t): \theta \leq t<2 \pi) \\
& =\operatorname{Sup}\left(\frac{1}{2 \pi I_{0}(k)} \exp (k \cos (t-\mu)): \theta \leq t<2 \pi\right)
\end{aligned}
$$

Normalizing this function with the constant $\mathrm{K}_{1}=\int_{0}^{2 \pi} g_{\Theta}(\theta) d \theta$ the pdf of the Rising Sun von Mises distribution (RSVM) is obtained.

$g_{c}(\theta)=\frac{\operatorname{Sup}\left(\frac{1}{2 \pi I_{0}(k)} \exp (k \cos (t-\mu)): \theta \leq t<2 \pi\right)}{\int_{0}^{2 \pi} g_{\Theta}(\theta) d \theta}$ 
The graphs of pdf of the von Mises, Rising Sun of von Mises and pdf of Rising Sun von Mises models are plotted here.

Fig. 3.1 Graph of von Mises pdf and the Rising Sun von Mises pdf

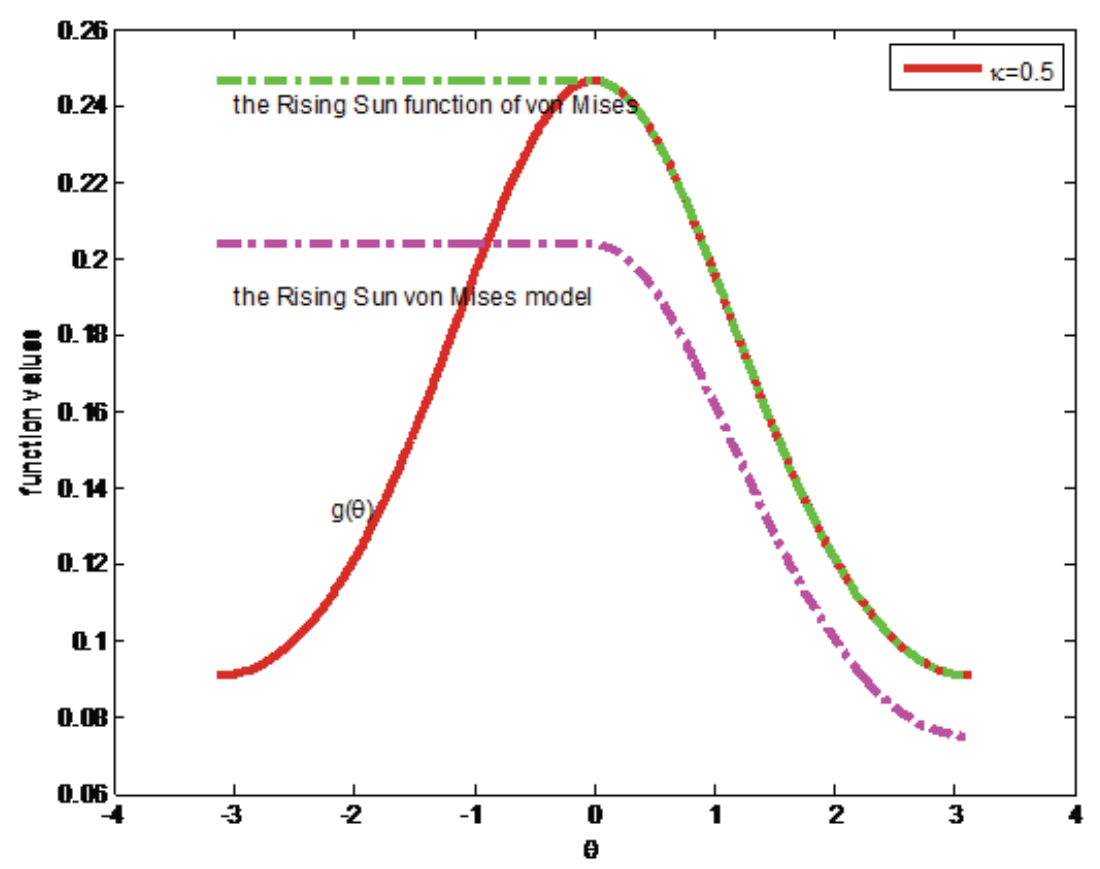

The Characteristic function of von Mises distribution

Since the distribution is symmetrical about $\mu$,

$$
\begin{aligned}
\bar{\beta}_{p} & =E[\sin p(\theta-\mu)] \\
\bar{\alpha}_{p} & =\frac{1}{2 \pi I_{0}(k)} \int_{0}^{2 \pi} \cos p(\theta-\mu) e^{k \cos (\theta-\mu)} d \theta \\
& =\frac{I_{p}(k)}{I_{0}(k)}
\end{aligned}
$$

where $I_{p}$ is the modified Bessels function of the first kind and order $p$. 
The modified $I_{p}$ is defined by $I_{p}(k)=\frac{1}{2 \pi} \int_{0}^{2 \pi} \cos p \theta e^{k \cos \theta} d \theta$

\section{The Rising Sun Wrapped Cauchy Model}

The Wrapped Cauchy distribution was introduced by Levy (1939) and has been studied by Wintner (1947). Mc Cullagh (1996) showed that Wrapped Cauchy distribution can be obtained by mapping Cauchy distribution onto the circle by the transformation $x \leftrightarrow 2 \tan ^{-1} x$.

The pdf of the Wrapped Cauchy distribution [Mardia and Jupp (2000)] is

$$
g(\theta)=\left(\frac{1}{2 \pi}\left(1+2 \sum_{p=1}^{\infty} \rho^{p} \cos p(\theta-\mu)\right)\right)
$$

where $\rho=\pi e^{-a}, a>0$ and $\theta \in[0,2 \pi)$

We shall denote this distribution by WC $(\mu, \rho)$. It follows from considering the real part of the geometric series $\sum_{p=1}^{\infty} \rho^{p} e^{-i p(\theta-\mu)}$ that reduces to

$$
g(\theta)=\frac{\left(1-\rho^{2}\right)}{2 \pi\left(1+\rho^{2}-2 \rho \cos (\theta-\mu)\right)}
$$

In particular, the mean direction is $\mu(\bmod 2 \pi)$ and the mean resultant length is $\rho$ and this distribution is unimodal and symmetric about $\mu$.

The Rising Sun function of the Wrapped Cauchy distribution is given by

$$
g_{\Theta}(\theta)=\sup \left(\frac{1}{2 \pi}\left(\frac{\left(1-\rho^{2}\right)}{\left(1+\rho^{2}-2 \rho \cos (t-\mu)\right)}\right): \theta \leq t<2 \pi\right)
$$


Normalizing this function with the constant $K_{2}=\int_{0}^{2 \pi} g_{\Theta}(\theta) d \theta$ the pdf of the Rising Sun Wrapped Cauchy distribution (RSWC) is obtained.

$$
g_{c}(\theta)=\frac{\sup \left(\frac{1}{2 \pi}\left(\frac{\left(1-\rho^{2}\right)}{\left(1+\rho^{2}-2 \rho \cos (t-\mu)\right)}\right): \theta \leq t<2 \pi\right)}{\int_{0}^{2 \pi} g_{\Theta}(\theta) d \theta}
$$

The graphs of pdf of the Wrapped Cauchy, Rising Sun of Wrapped Cauchy and pdf of Rising Sun Wrapped Cauchy models are plotted here. 
Fig. 4.1 Graph of the Wrapped Cauchy pdf and the Rising Sun Wrapped Cauchy pdf

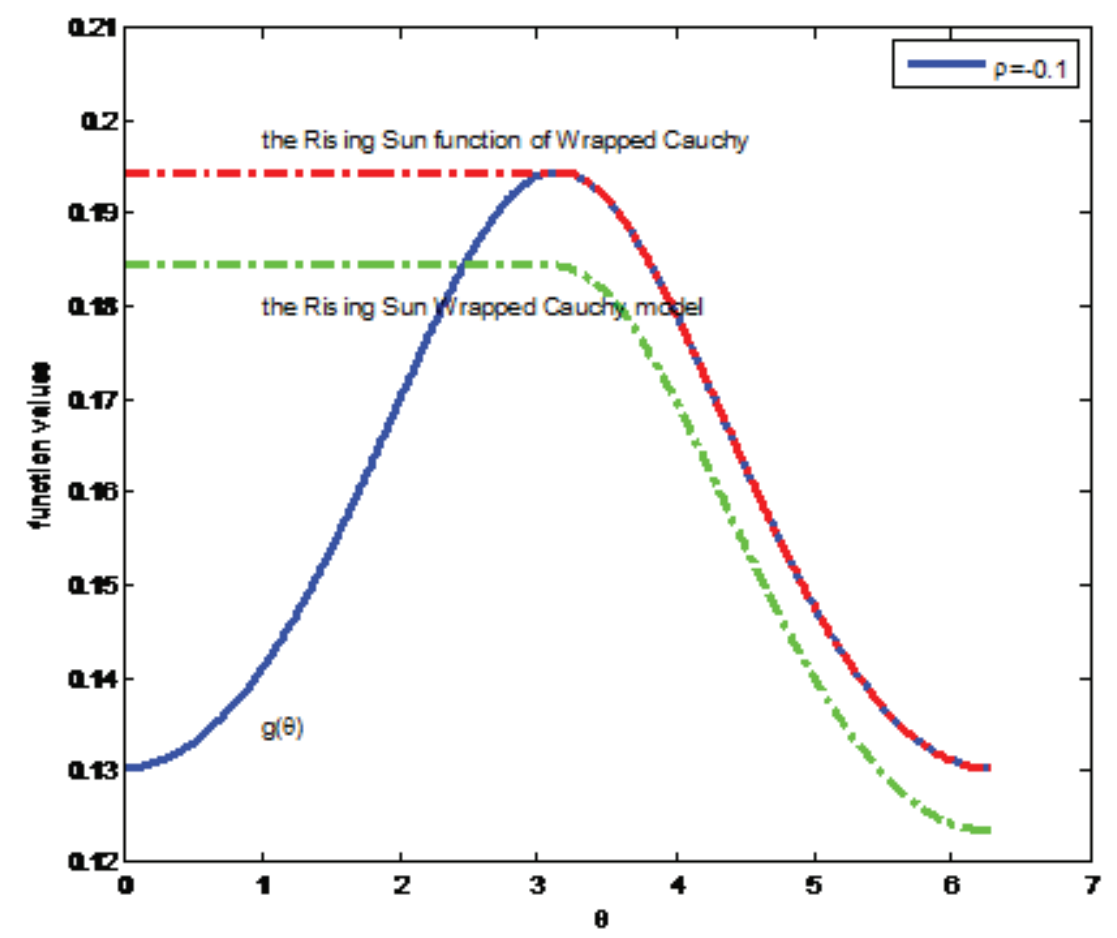

5. The Population Characteristics of New Rising Sun Circular Models

The characteristic function of a circular model with probability density function $g(\theta)$ is defined as $\varphi_{p}(\theta)=\int_{0}^{2 \pi} e^{i p \theta} g(\theta) d \theta, p \in Z$. Ramabhadra Sarma et al $(2009 ; 2011)$ derived the characteristic functions of some new wrapped models and Phani et al (2012) also presented the characteristic function of circular model induced by inverse stereographic projection.

\section{The Characteristic function of the Rising Sun circular model is derived here under}

Theorem 5.1: Let $g$ and $\phi_{p}$ be the pdf and the characteristic function of a circular model and $\theta_{1}$ be the mode of $g$. Then the characteristic function of the corresponding Rising Sun circular model with pdf $g_{c}$ is 
$\varphi_{\Theta}(p)=\left\{\begin{array}{c}1 \text { for } p=0 \\ \frac{\phi_{p}(\theta)}{k}+\frac{g\left(\theta_{1}\right)}{k p} i\left(1-e^{i p \theta_{1}}\right)-\frac{1}{k} \int_{0}^{\theta_{1}} e^{i p \theta} g(\theta) d \theta \text { for } p \neq 0\end{array}\right.$

where $g_{\Theta}$ is the Rising Sun function of $g$, and $k=\int_{0}^{2 \pi} g_{\Theta}(\theta) d \theta$

Proof: The pdf of the Rising Sun circular Model is

$$
g_{c}(\theta)=\frac{1}{k} g_{\Theta}(\theta)
$$

For $p \neq 0$

The characteristic function of the Rising Sun circular model is

$$
\begin{aligned}
\varphi_{\Theta}(p)=\int_{0}^{2 \pi} e^{i p \theta} g_{c}(\theta) d \theta=\frac{1}{k} \int_{0}^{2 \pi} e^{i p \theta} g_{\Theta}(\theta) d \theta \\
\varphi_{\Theta}(p)=\frac{1}{k}\left[\int_{0}^{\theta_{1}} e^{i p \theta} g_{\Theta}(\theta) d \theta+\int_{\theta_{1}}^{2 \pi} e^{i p \theta} g_{\Theta}(\theta) d \theta\right] \\
=\frac{1}{k}\left[\int_{0}^{\theta_{1}} e^{i p \theta} g\left(\theta_{1}\right) d \theta+\int_{\theta_{1}}^{2 \pi} e^{i p \theta} g(\theta) d \theta\right] \\
=\frac{g\left(\theta_{1}\right)}{k}\left(\frac{-i e^{i p \theta}}{p}\right)_{0}^{\theta_{1}}+\frac{1}{k} \phi_{p}(\theta)-\frac{1}{k} \int_{0}^{\theta_{1}} e^{i p \theta} g(\theta) d \theta
\end{aligned}
$$

by Theorem $4.3 g_{\Theta}(\theta)=g\left(\theta_{1}\right)$ for $\theta \leq \theta_{1}$

$$
=g(\theta) \text { for } \theta_{1}<\theta
$$

$$
\text { for } p=0, \varphi_{\Theta}(0)=\frac{1}{k} \int_{0}^{2 \pi} g_{\Theta}(\theta) d \theta=1
$$


Hence $\varphi_{\Theta}(p)=\left\{\begin{array}{c}1 \text { for } p=0 \\ \frac{\phi_{p}(\theta)}{k}+\frac{g\left(\theta_{1}\right)}{k p} i\left(1-e^{i p \theta_{1}}\right)-\frac{1}{k} \int_{0}^{\theta_{\theta}} e^{i p \theta} g(\theta) d \theta \text { for } p \neq 0\end{array}\right.$

The graphs of the characteristic functions of new Rising Sun circular models are presented here.

Fig. 5.1 Graph of the Characteristic Function of the Rising Sun von Mises distribution

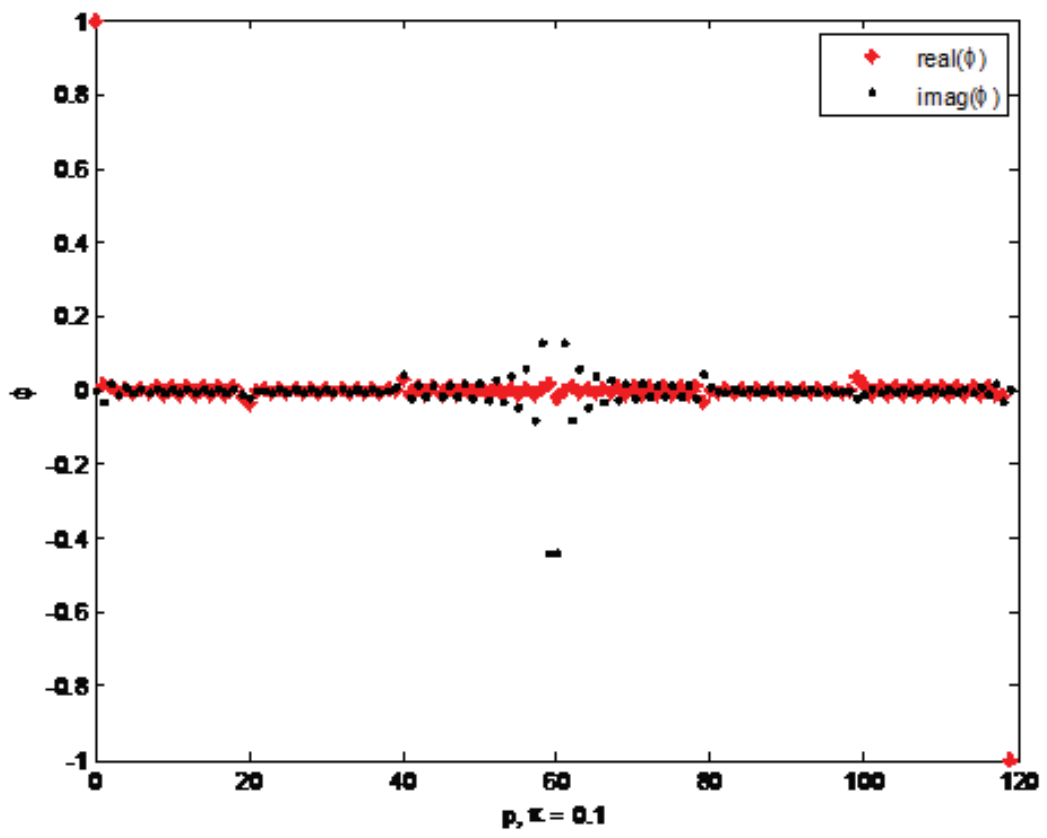


Fig 5.2 Graph of the Characteristic Function of the Rising Sun Wrapped Cauchy distribution

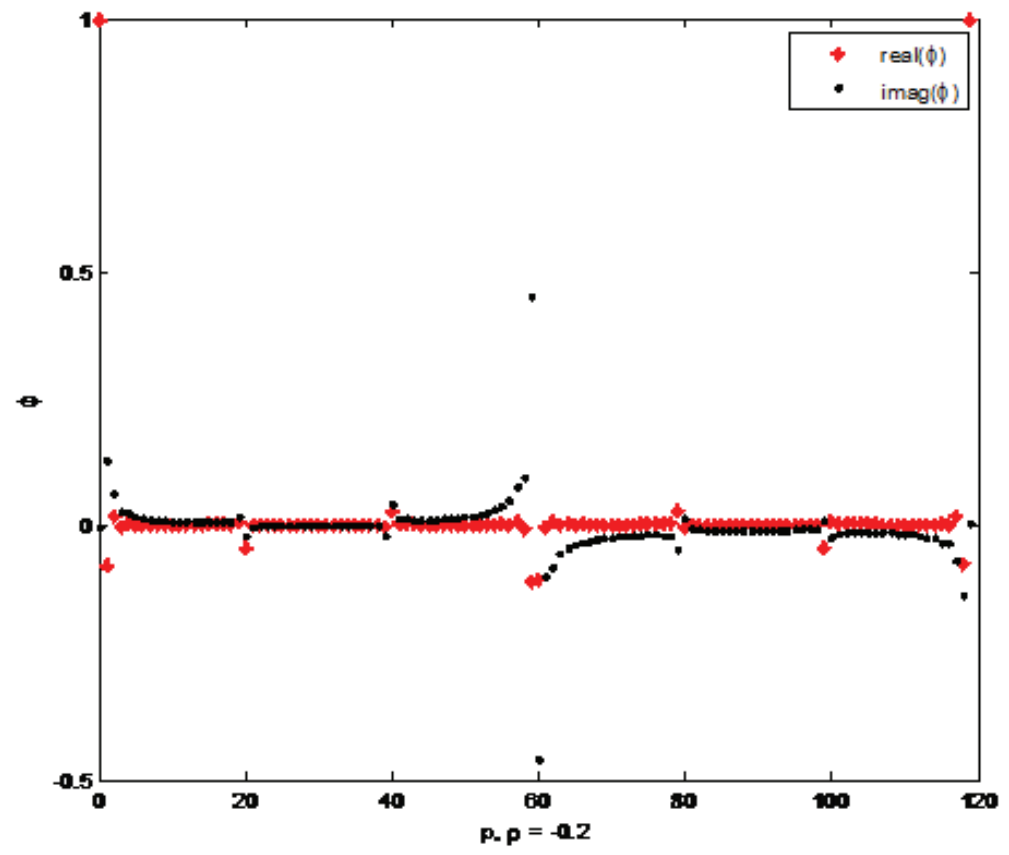

To study the characteristics of the circular models, the trigonometric moments which are the real and imaginary parts of the characteristic function are required. As the pdf of the newly constructed Rising Sun circular models are not in closed form, the values of the characteristic functions are evaluated using numerical methods in MATLAB 7. The characteristics for the Rising Sun von Mises and the Rising Sun Wrapped Cauchy models are also based on their respective trigonometric moments. These can be expressed in terms of trigonometric moments $\alpha_{p}$ and $\beta_{p}$. [Mardia and Jupp (2000)] and are presented in tables 1 and 2. 
Table 1: The Characteristics of the Rising Sun von Mises distribution

\begin{tabular}{|c|c|c|c|c|c|}
\hline & $k=0.1$ & $k=0.2$ & $k=0.5$ & $k=0.75$ & $k=1$ \\
\hline $\begin{array}{ll}\text { Trigonometric } & \alpha_{1} \\
\text { Moments } & \alpha_{2} \\
& \\
& \beta_{1}\end{array}$ & $\begin{array}{l}0.0162 \\
0.0080 \\
-0.0312 \\
0.0203\end{array}$ & $\begin{array}{l}0.0382 \\
0.0088 \\
-0.0610 \\
0.0382\end{array}$ & $\begin{array}{l}0.0899 \\
0.0165 \\
-0.1418 \\
0.0801\end{array}$ & $\begin{array}{r}0.1190 \\
0.0262 \\
-0.1984 \\
0.1030\end{array}$ & $\begin{array}{l}0.1383 \\
0.0371 \\
-0.2458 \\
0.1174\end{array}$ \\
\hline Mean direction $\quad \mu_{1}$ & -1.0920 & -1.0117 & -1.0057 & -1.0305 & -1.0582 \\
\hline $\begin{array}{l}\text { Resultant length } \quad \rho \\
\end{array}$ & $\begin{array}{l}0.0351 \\
0.0218\end{array}$ & $\begin{array}{l}0.0719 \\
0.0392\end{array}$ & $\begin{array}{l}0.1679 \\
0.0818\end{array}$ & $\begin{array}{l}0.2314 \\
0.1063\end{array}$ & $\begin{array}{l}0.2821 \\
0.1232\end{array}$ \\
\hline Circular Variance $V_{o}$ & 0.9649 & 0.9281 & 0.8321 & 0.7686 & 0.7179 \\
\hline $\begin{array}{ll}\text { Central } & \alpha_{1}^{*} \\
\text { Trigonometric } & \alpha_{2}^{*} \\
\text { Moments } & \beta_{1}^{*} \\
& \\
& \beta_{2}^{*}\end{array}$ & $\begin{array}{l}0.0351 \\
-0.0212 \\
0 \\
-0.0051\end{array}$ & $\begin{array}{l}0.0719 \\
-0.0382 \\
0 \\
-0.0088\end{array}$ & $\begin{array}{c}0.1679 \\
-0.0795 \\
0 \\
-0.0193\end{array}$ & $\begin{array}{l}0.2314 \\
-0.1032 \\
0 \\
-0.0254\end{array}$ & $\begin{array}{l}0.2821 \\
-0.1196 \\
0 \\
-0.0293\end{array}$ \\
\hline Skewness & -0.0054 & -0.0098 & -0.0254 & -0.0376 & -0.0481 \\
\hline Kurtosis $\quad \gamma_{2}^{o}$ & -0.0228 & -0.0444 & -0.1159 & -0.1796 & -0.2444 \\
\hline $\begin{array}{l}\text { Circular } \\
\text { Standard deviation } \sigma_{o}\end{array}$ & $\begin{array}{l}2.5881 \\
2.7660\end{array}$ & $\begin{array}{c}2.2944 \\
2.5450\end{array}$ & $\begin{array}{l}1.8892 \\
2.2378\end{array}$ & $\begin{array}{l}1.7109 \\
2.1172\end{array}$ & $\begin{array}{l}1.5910 \\
2.0466\end{array}$ \\
\hline
\end{tabular}


ON RISING SUN VON MISES AND RISING SUN WRAPPED CAUCHY ...

Table 2: Characteristics of the Rising Sun Wrapped Cauchy distribution

\begin{tabular}{|c|c|c|c|c|}
\hline & $\rho=-0.2$ & $\rho=-0.1$ & $\rho=-0.25$ & $\rho=-0.4$ \\
\hline $\begin{array}{cc}\text { Trigonometric } & \alpha_{1} \\
\text { Moments } & \alpha_{2} \\
& \beta_{1} \\
& \beta_{2}\end{array}$ & $\begin{array}{l}-0.0743 \\
0.0212 \\
0.1335 \\
0.0662\end{array}$ & $\begin{array}{c}-0.0381 \\
0.0111 \\
0.0653 \\
0.0380\end{array}$ & $\begin{array}{l}-0.0885 \\
0.0281 \\
0.1685 \\
0.0764\end{array}$ & $\begin{array}{l}-0.1160 \\
0.0515 \\
0.1685 \\
0.0764\end{array}$ \\
\hline Mean direction $\quad \mu_{1}$ & -1.0632 & -1.0424 & -1.0870 & -1.1712 \\
\hline Resultant length $\rho$ & $\begin{array}{l}0.1528 \\
0.0695\end{array}$ & $\begin{array}{l}0.0756 \\
0.0396\end{array}$ & $\begin{array}{l}0.1900 \\
0.0815\end{array}$ & $\begin{array}{l}0.2981 \\
0.1051\end{array}$ \\
\hline Circular Variance $V_{o}$ & 0.8472 & 0.9244 & 0.8097 & 0.7019 \\
\hline $\begin{array}{ll}\text { Central } & \alpha_{1}^{*} \\
\text { Trigonometric } & \alpha_{2}^{*} \\
\text { Moments } & \beta_{1}^{*} \\
& \\
& \beta_{2}^{*}\end{array}$ & $\begin{array}{c}0.1528 \\
-0.0674 \\
0 \\
-0.0169\end{array}$ & $\begin{array}{c}0.0756 \\
-0.0385 \\
0 \\
-0.0090\end{array}$ & $\begin{array}{c}0.1903 \\
-0.0789 \\
0 \\
-0.0202\end{array}$ & $\begin{array}{c}0.2981 \\
-0.1016 \\
0 \\
-0.0270\end{array}$ \\
\hline Skewness & -0.0216 & -0.0102 & -0.0277 & -0.0459 \\
\hline Kurtosis & -0.0947 & -0.0451 & -0.1224 & -0.2222 \\
\hline $\begin{array}{l}\text { Circular } \\
\text { Standard deviation } \sigma_{o}\end{array}$ & $\begin{array}{l}1.9384 \\
2.3093\end{array}$ & $\begin{array}{l}2.2728 \\
2.5415\end{array}$ & 1.82162 .2395 & $\begin{array}{l}1.5558 \\
2.1226\end{array}$ \\
\hline
\end{tabular}




\section{A.J.V. RADHIKA, S.V.S.GIRIJA, A.V. DATTATREYA RAO}

\section{REFERENCES}

1. Dattatreya Rao, A.V., Ramabhadra Sarma, I. and Girija, S.V.S. (2007). On Wrapped Version of Some Life Testing Models, Comm Statist., - Theor.Meth. 36, 11, pp.2027-2035.

2. Girija, S.V.S. (2010).Construction of New Circular Models, (monograph) VDM VERLAG, Germany. ISBN 978-3-639-27939-9.

3. Girija S.V.S, Radhika A. J. V. and Dattatreya Rao A.V, (2013), On Bimodal Offset Cauchy Distribution, Journal of the Applied Mathematics, Statistics and Informatics (JAMSI), Vol.9, No.1.

4. Ivan Markovi'c and Ivan Petrovi'c, (2010). Applying von Mises Distribution to Microphone Array Probabilistic Sensor Modelling, Robotics (ISR), 41st International Symposium on and 2010 6th German Conference on Robotics (ROBOTIK).

5. Jammalamadaka, S.R. and Sen Gupta, A. (2001). Topics in Circular Statistics, World Scientific Press, Singapore.

6. Levy, P. (1939). L'addition des variables aleatories definies sur une circonference, Bull.Soc.Math., France, 67, pp $1-41$.

7. Mardia, K.V. and Jupp, P.E. (2000). Directional Statistics, John Wiley, Chic ester.

8. Mardia, K. V., Gareth Hughes and Charles C. Taylor,(2008), A Multivariate von Mises Distribution with Applications to Bioinformatics, The Canadian Journal of Statistics, vol 36, no. 1, pp. 99 - 109.

9. Mc Cullagh,P.(1996). Mobius transformation and Cauchy parameter estimation. Ann.Statist., 24, pp $787-808$.

10. Phani, Y., Girija S.V.S. and Dattatreya Rao A.V., (2012). Circular Model Induced by Inverse Stereographic Projection On Extreme-Value Distribution, IRACST - Engineering Science and Technology: An International Journal (ESTIJ), ISSN: 2250-3498, Vol.2, No. 5, pp. 881 - 888.

11. Radhika A J V, Girija S.V.S. and Dattatreya Rao A.V. (2013), On Univariate Offset Pearson Type II Model - Application To Live Data, International Journal of Mathematics and Statistics Studies, Vol.1, No. 1, pp.1-9

12. Ramabhadra Sarma, I., Dattatreya Rao, A.V. and Girija, S.V.S., (2009). On Characteristic Functions of Wrapped Half Logistic and Binormal Distributions, International Journal of Statistics and Systems, 4(1), 33-45.

13. Ramabhadra Sarma, I., Dattatreya Rao, A.V. and Girija, S.V.S., (2011). On Characteristic Functions of Wrapped Lognormal and Weibull Distributions, Journal of Statistical Computation and Simulation Vol. 81(5), 579-589.

14. Singh, H., Hnizdo, V. and Demchuk, E. (2002). Probabilistic model for two dependent circular variables. Biometrika, 89, 719-723.

15. Van Rooij, A.C.M. and Schikof, W.H. (1982). A Second Course on Real Functions, Cambridge University Press, Cambridge.

16. von Mises, R. (1918). Uber die "Ganzzahligkeit" der Atomgewichte and verwandte Frogmen. Phys.Z., 19, 490 - 500.

17. Wintner, A. (1947). On the shape of the angular case of Cauchy's distribution curves. Ann. Math. Statist., 18, 589-593. 
ACKNOWLEDGEMENT: Authors would like to thank UGC, New Delhi, India for offering financial assistance to carry out the project under the head of Major Research Project no. F 41-785/2012 (SR) dt. 17-07-2012.

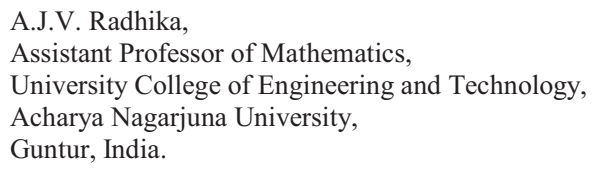

S.V.S.Girija,

Associate Professor of Mathematics,

Hindu College,

Guntur, India.

A.V. Dattatreya Rao,

Professor of Statistics,

Acharya Nagarjuna University,

Guntur, India.

Received October 2013 